\title{
The influence of hla genotype on the development of metal hypersensitivity following joint replacement
}

\section{David Langton ( $\sim$ Djlangton22@doctors.org.uk)}

ExplantLab and NHS

\section{Rohan Bhalekar}

ExplantLab

\section{Thomas Joyce}

Newcastle University

Benjamin Wainwright

Yale NUS College

\section{Matthew Nargol}

ExplantLab

\section{Nish Shyam}

ExplantLab https://orcid.org/0000-0002-8420-9770

\section{Benedicte Lie}

3Department of Medical Genetics, University of Oslo

\section{Moreica Pabbruwe}

Royal Perth Hospital

\section{Alan Stewart \\ Susan Waller}

University Hospital of North Tees

\section{Shonali Natu}

University Hospital of North Tees

\section{Renee Ren}

Hospital for Special Surgery

\section{Rachelle Hornick}

Hospital for Special Surgery

\section{Rebecca Darlay}

Newcastle University

\section{Edwin Su}

Hospital for Special Surgery

\section{Antoni Nargol}

University Hospital of North Tees 


\section{Article}

Keywords: joint, implant, responses, patients, material

Posted Date: October 29th, 2021

DOI: https://doi.org/10.21203/rs.3.rs-918228/v1

License: (c) (i) This work is licensed under a Creative Commons Attribution 4.0 International License. Read Full License

Version of Record: A version of this preprint was published at Communications Medicine on June 24th, 2022. See the published version at https://doi.org/10.1038/s43856-022-00137-0. 


\title{
THE INFLUENCE OF HLA GENOTYPE ON THE DEVELOPMENT OF METAL HYPERSENSITIVITY FOLLOWING JOINT REPLACEMENT
}

\author{
David J Langton ${ }^{1}$ \\ Rohan M Bhalekar ${ }^{1}$ \\ Thomas J Joyce ${ }^{2}$ \\ Stephen P Rushton ${ }^{2}$ \\ Benjamin J Wainwright ${ }^{3}$ \\ Matthew E Nargol ${ }^{1}$ \\ Nish Shyam ${ }^{1}$ \\ Benedicte Lie ${ }^{4}$ \\ Moreica Pabbruwe ${ }^{5}$ \\ Alan Stewart ${ }^{6}$ \\ Susan Waller ${ }^{7}$ \\ Shonali Natu ${ }^{7}$ \\ Renne $\operatorname{Ren}^{8}$ \\ Rachelle Hornick ${ }^{8}$ \\ Rebecca Darlay ${ }^{2}$ \\ Edwin $\mathrm{Su}^{8}$ \\ Antoni VF Nargol ${ }^{7}$
}

1. ExplantLab, The Biosphere, Newcastle Helix, Newcastle-upon-Tyne, England.

2. Newcastle University, Newcastle-upon-Tyne, England.

3. Yale-NUS College, 16 College Avenue West, 138527, Singapore

4. Department of Medical Genetics, University of Oslo and Oslo University Hospital.

5. Royal Perth Hospital, Perth Australia

6. School of Medicine, University of St Andrews

7. University Hospital of North Tees, Stockton, England

8. Hospital for Special Surgery, New York, United States 
Joint replacement surgery provides pain relief and restoration of mobility for millions of patients around the world each year.[1] However, the release of wear debris from implant surfaces can limit the lifespan of a prosthesis through the promotion of inflammatory responses.[2] Implants must therefore be constructed from materials with sufficient durability and biocompatibility. One such material is cobalt chrome alloy, which is used in the majority of joint replacements. [3] Unfortunately, it is recognised that some patients develop lymphocyte mediated delayed type hypersensitivity (DTH) responses to this material,[4] a response which may result in extensive bone and soft tissue destruction.[5] A genetic predisposition to DTH has been proposed[6], though specific genes have yet to be identified, or the effects quantified. Here we show that variation in HLA class II genotype influences an individual's susceptibility to DTH. HLA-DQ haplotypes encoding peptide binding grooves with greater affinity for the $\mathbf{N}$ terminal peptide sequence of albumin (containing two recognised metal binding sites) confer a greater risk of DTH. We describe the development and validation of a machine learning algorithm to investigate the possibility that a patient's genotype and basic clinical parameters may be used to predict DTH. Incorporating this novel finding, gradient boosted survival analysis machine learning models were trained and validated using results from 606 patients from three international units. These models were assessed using Uno's c-index, time-dependent AUROCs, and integrated calibration index performance statistics. At present, there are no tests in widespread clinical use which use a patient's genetic profile to guide implant selection or inform post-operative management. The algorithm described herein may address this issue. 


\section{Background}

Hip joint replacement surgery (hip arthroplasty) has proven to be extremely successful in the treatment of end stage hip arthritis. As a result, there are now approximately 2 million hip arthroplasties carried out in OECD countries alone.[1]

Conventional total hip replacements (THRs) are composed of a metal femoral head which articulates against a polyethylene (plastic) cup.[2] The lifespan of these so called metal on polyethylene (MoP) prostheses may be limited in younger, more active patients. This is because during activities of daily living, the harder metal head wears away plastic cup. The release of greater amounts of wear debris over time increases the probability of a macrophage driven, adverse immune response developing in the periprosthetic tissue.[2] The result of this is "wear induced osteolysis", in which the bony architecture surrounding the implant becomes compromised and the component/components loosen.[7] When a prosthesis must be replaced it is deemed to have "failed"; revision surgery must be undertaken and a new device implanted.

Metal on metal (MoM) hip prostheses were reintroduced at the turn of the century to address this problem.[8] In MoM hips, the softer polyethylene cup is replaced by a wear resistant cobalt chrome ( $\mathrm{CoCr}$ ) cup (figure 1). It was hoped that the use of harder wearing $\mathrm{CoCr}$ bearing surfaces would lead to a reduction in the volume of wear debris, thus improving implant longevity. The initial early success of the Birmingham Hip Resurfacing (BHR) in young males[8], saw a rapid expansion in the market, with ever widening patient eligibility criteria and several new prostheses released from competing manufacturers.[9]

Unfortunately, it became apparent some patients were beginning to experience problems.[10] Symptoms developed a number of years post surgery, typically with new onset groin pain, aggravated by straight leg raise. Often, large, sterile fluid collections developed in the joint. Histopathological examination of tissues excised at revision surgery identified a macrophagic infiltration - as previously encountered in MoP prostheses - but frequently this macrophage 
response was accompanied by a perivascular T lymphocyte infiltrate. In severe cases, these perivascular cuffs expand in circumference, coalesce, leading to the formation of germinal centres and destruction of the synovial surface.[11] In a seminal paper, Willert et al coined the term "aseptic lymphocyte dominated vasculitis association lesion" (ALVAL) to describe these histopathological features.[4] ALVAL can frequently be associated with destruction of local tissues including bone, muscle and neurovascular structures.[12] These lesions are progressive, and if revision surgery is delayed, the incidence of major post operative complications increases.[13] Reimplantation with CoCr components leads to rapid recurrence of symptoms.[14] The overall clinical and histopathological picture is indicative of a delayed type hypersensitivity (DTH) response to the metal debris shed from the prostheses.[4] Note: In this paper we use the terms DTH and ALVAL interchangeably, with ALVAL the preferred term when referring specifically to MoM hip patients in the current study.

\section{Figure 1}

MoM hip components are manufactured from standard, medical grade $\mathrm{CoCr}$ alloy (ASTM-75 or ASTM-1537), which is composed of approximately $65 \% \mathrm{Co}$ and $30 \% \mathrm{Cr}$ by weight. Explanted prostheses can be reverse engineered using coordinate measuring machines to quantify the volumetric material loss through wear and corrosion (shown in red below). Serum or whole blood Co and $\mathrm{Cr}$ concentrations provide a reliable in-vivo surrogate measure of the rate of material loss from an implant.[15] 


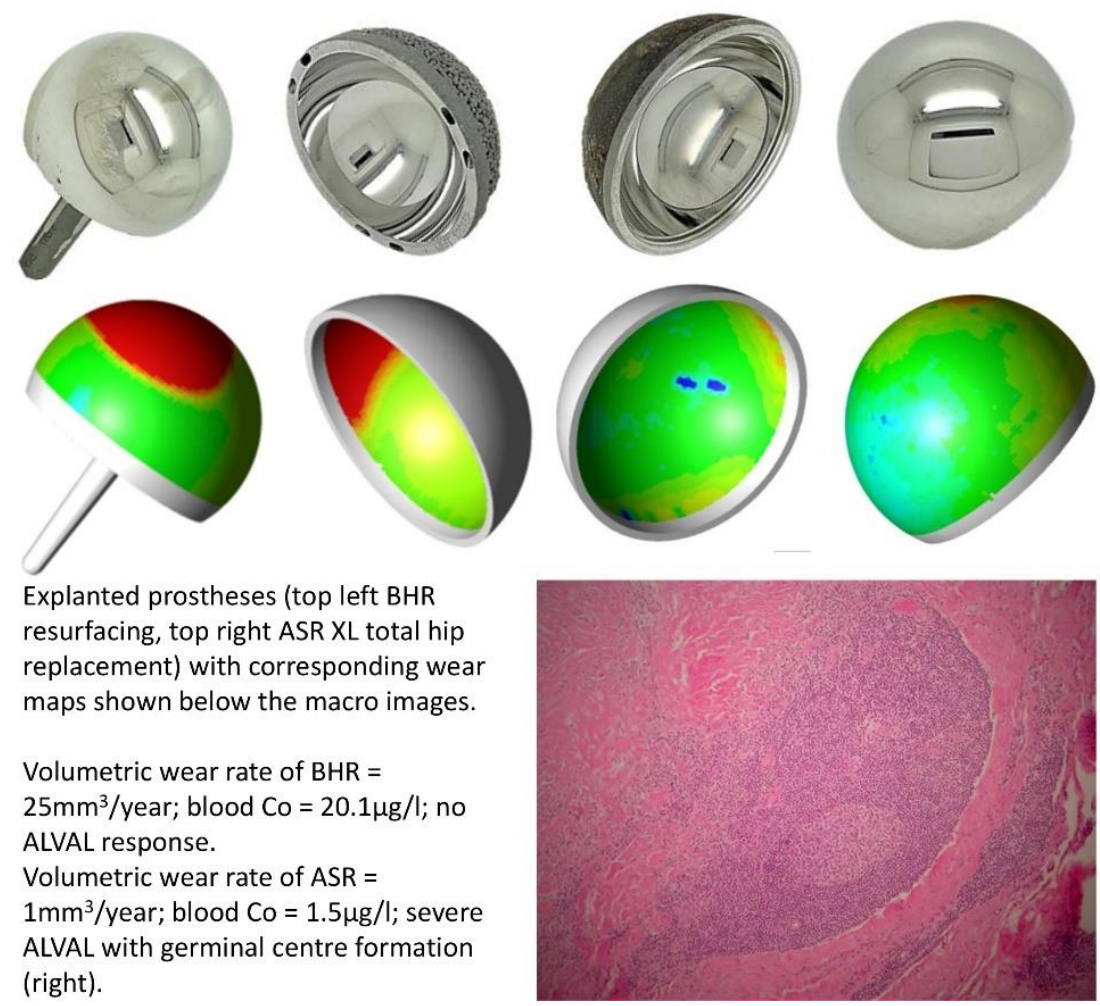

Studies have shown that the risk of tissue damage is increased when prostheses shed greater volumes of metal debris.[16] Consequently, in 2012, the Medicines and Healthcare Products Regulatory Agency (MHRA, UK) issued an alert regarding the management of patients with MoM implants. In it they recommended the monitoring of metal concentrations in blood, establishing a threshold of 7 micrograms per litre $(\mu \mathrm{g} / \mathrm{l})$ of $\mathrm{Co}$ or $\mathrm{Cr}$ as an indicator of an adverse tissue reaction. Although these guidelines were based on a small study involving only 26 patients[17], the guidance has not been substantially modified since its first release.[18] However, patients display varying tolerances to metallic debris[19](figure 1) and women in particular, appear to be at greater risk of developing hypersensitivity.[20]

\section{Delayed type hypersensitivity}

There are two phases of DTH: sensitisation and elicitation. During the sensitisation phase, antigen presenting cells (APCs) take up, process and display an antigen (figure 2). APCs migrate to regional lymph nodes where the displayed antigen may activate T4 cells and the production of memory $\mathrm{T}$ 
cells, which migrate to the original site. In the elicitation phase, a subsequent exposure to the antigen leads to its re-presentation to memory $\mathrm{T}$ cells with the release of $\mathrm{T}$ cell chemokines and cytokines such as interferon gamma, which enhance the inflammatory response.

\section{Figure 2.}

1. The $\mathrm{N}$ terminal sequence (NTS) of albumin contains two recognised metal binding sites.

2. Endogenous (self-proteins, including albumin) and exogenous (pathogens/ingested substances) proteins are ingested by APCs, which include macrophages and dendritic cells (purple).

3. The ingested proteins are acidified in the lysosomes of the APCs(29), where peptidases act to section the original proteins into their shorter, constituent peptides. These peptides compete for the binding groove of class II MHC complexes (red).

4. If a peptide forms a stable complex with the MHC molecule, the resulting MHC:peptide complex is transported to the cell surface

5. Self-peptides held in the binding groove of an MHC molecule can form complexes with metal ions (dark red sphere) released from implant surfaces (broken dark red arrow) to form haptens.

6. The MHC:peptide:metal complex may activate T4 lymphocytes resulting in sensitisation. This will happen if the lymphocyte has been activated via pattern recognition receptors (PRRs), which can happen due to local cellular damage through metal toxicity (broken red arrow). 


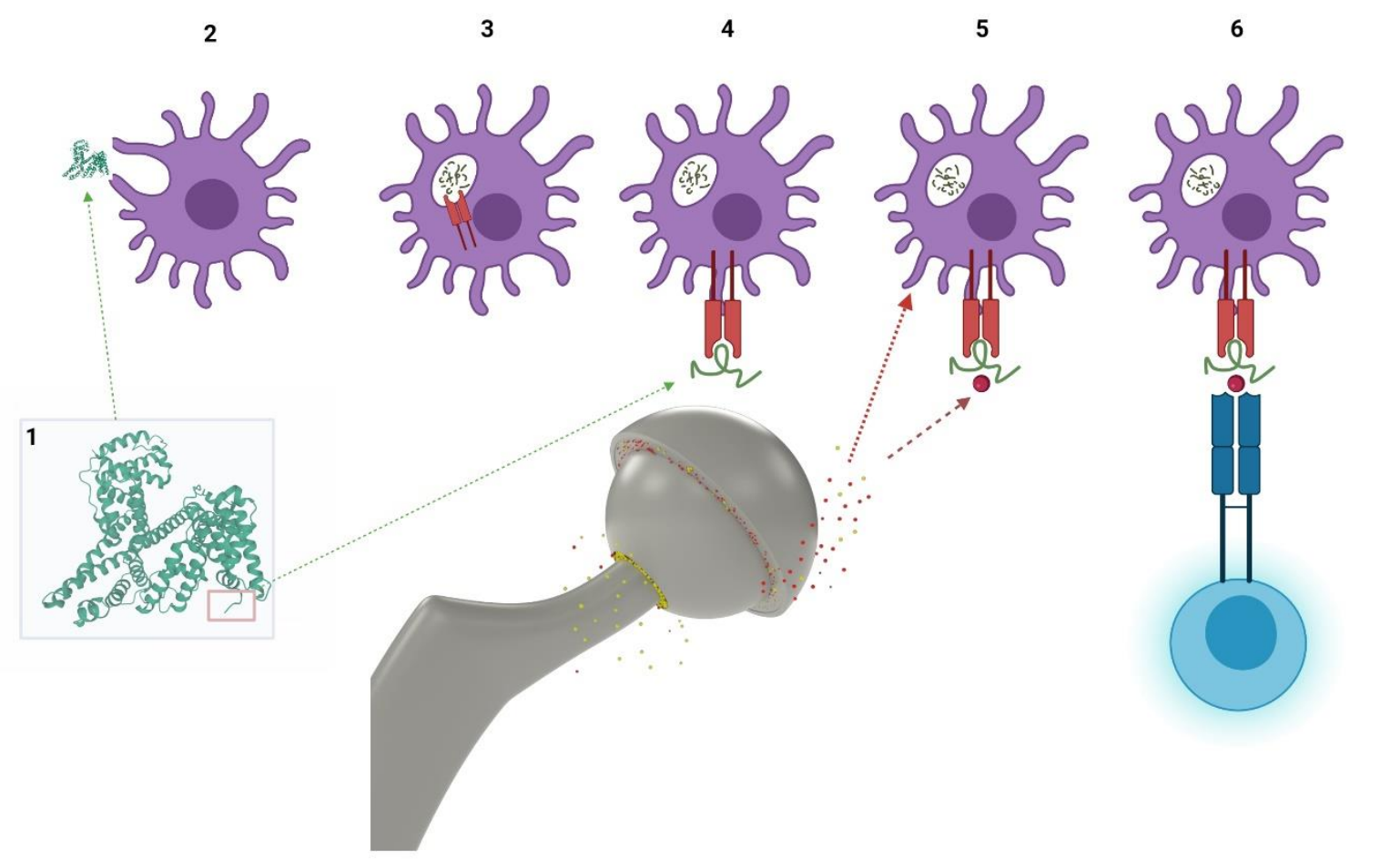

A critical factor in the development of DTH, therefore, is the presentation of a specific peptide/antigen at the peptide binding groove of an APC. This is a competitive process, described in figure 3.

\section{Figure 3.}

The HLA-DQ molecule is an $\alpha \beta$ heterodimer of MHC class type II. The three dimensional shape of the peptide binding groove is formed by the combination of $\alpha$ and $\beta$ chains, which are genetically encoded by the HLA-DQA1 and HLA-DQB1 alleles respectively.[21] The structure of the peptide binding groove determines which peptides (foreign or self) are presented at the cell's surface[21], as is shown in the schematic below. As an example, in coeliac disease, patients possess HLA alleles 
which encode for peptide binding grooves with structures suited to the presentation of gluten derived peptides.[22]

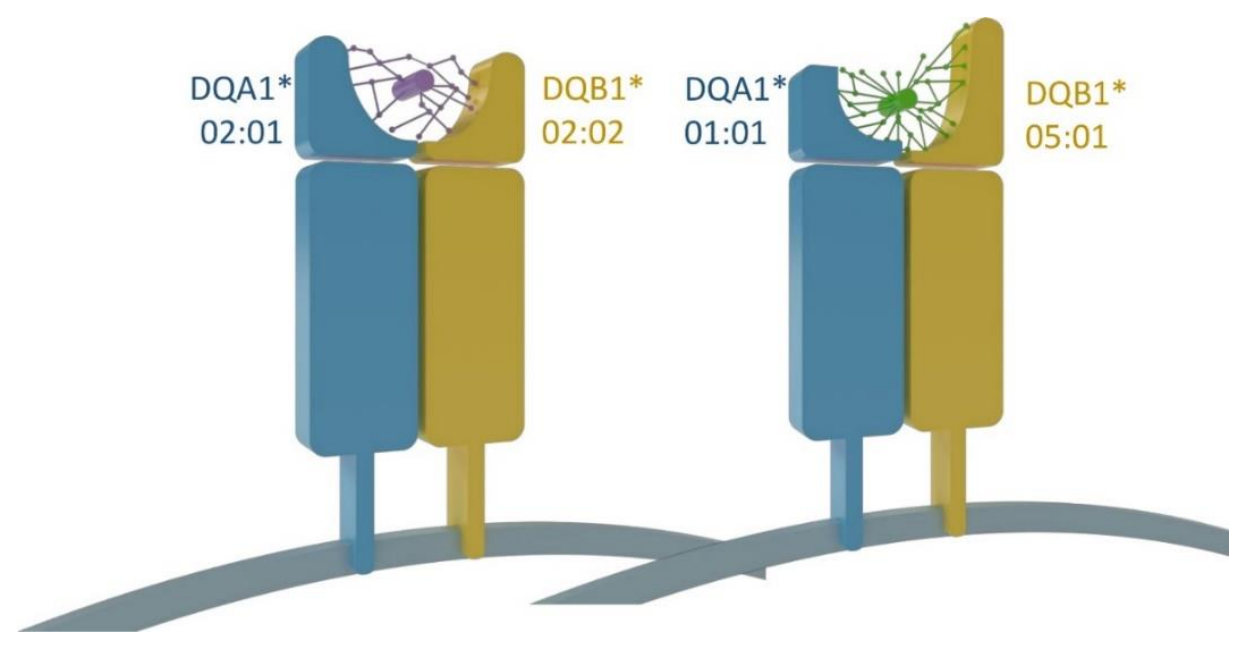

Metals are capable of provoking a variety of T cell mediated, HLA linked diseases, such as chronic beryllium disease[23], Co hard metal lung disease[24], and contact hypersensitivities[25]. Three pathogenic mechanisms have been described:

1) Self peptides held in the binding groove of an $\mathrm{MHC}$ molecule form complexes with metal ions, with the resulting complexes acting as antigens.[26]

2) T cells recognize metal induced changes to the MHC molecule itself.[27]

3) Metals directly affect the processing of self-peptides, resulting in T cells reactive to cryptic selfpeptides.[28]

Which mechanism may be most important in ALVAL? Previous research and clinical experience indicated that the first mechanism would most likely be involved in the initiation of the ALVAL response, and that the $\mathrm{N}$ terminal sequence (NTS) of albumin was the prime candidate peptide sequence to investigate. We therefore hypothesized that individuals developing ALVAL may have 
greater frequencies of (HLA gene encoded) peptide binding grooves with greater affinities for the NTS of albumin.

To carry out this investigation, we accessed a large database which was set up over a decade ago as part of an ethically approved study (IRAS14119) at site 1 (United Kingdom). This data set, comprising over 500 MoM hip revisions, includes pre revision blood metal ion concentrations, semiquantitative grading scores of tissues excised at revision surgery and volumetric wear analysis of the explanted components. We identified patients who had developed the most aggressive ALVAL responses to the lowest wearing prostheses (patient group 1) and compared their HLA genotypes to those of: a group of patients who remained asymptomatic at a minimum of ten years following their surgery (group 2); a group who developed ALVAL in response to prostheses wearing at high rates (group 3); and a group of patients who had experienced joint failure without signs of ALVAL (group 4). Using validated in silico techniques, we converted the HLA alleles most strongly linked to ALVAL to NTS binding affinities, then tested the possibility that a machine learning algorithm may be developed for clinical use to predict the risk of ALVAL developing in the preoperative (for appropriate implant selection) and the post-operative environment (to improve patient follow up regimes). This algorithm was trained and validated using data from two independent, international units.

\section{Results}

\section{Part 1: Investigation of genetic associations through comparison of extreme phenotype groups}

\section{(unit 1)}

There was a response rate of around $60 \%$ in each of the four patient groups, resulting in a total of 161 patients who gave saliva or buccal samples for DNA analysis. Patient details and class II haplotype distributions can be seen in extended data table 1 and supplementary table 1 . There was a bias towards female sex, increased age and THRs in patients developing ALVAL in response to 
lower wear. The strongest signals were found with two haplotypes, which had opposing associations with ALVAL. The dominant, significant positive association was seen with DQA1*02:01, DQB1*02:02 and DRB1*07:01. These alleles were increased across all phenotypic subtypes. The alleles were in strong linkage disequilibrium and occurred on one associated haplotype. A protective effect was seen with the alleles DQA1*01:01, DQB1*05:01 and DRB1*01:01. These alleles, also in strong linkage disequilibrium and occurring on one associated haplotype, were found in significantly higher frequencies in patients without ALVAL. Class I HLA allele distributions did not differ between the groups.

Part 2: In silico analysis of peptide-HLA class II binding affinity and Cox proportional hazards modelling (unit 1)

DQA1*02:01-DQB1*02:02, the haplotype with the strongest positive association to ALVAL, exhibited the strongest binding affinity to the NTS of albumin. DQA $1 * 01: 01-D Q B 1 * 05: 01$, the commonly occurring haplotype with the strongest negative association with ALVAL, exhibited one of the weakest binding affinities in the dataset (rank 15 out of 17 haplotypes). Cox proportional hazards modelling, incorporating NTS binding affinities as a continuous measure of DQ haplotype, demonstrated that pre-revision blood Co and Cr concentrations, female sex and greater NTS binding affinity were significantly associated with increased risk of early ALVAL associated failure. These models were consistent using different thresholds of ALVAL (mild and above versus moderate and above (extended data table 2). No relationship was identified between prosthetic failure and the binding affinity values derived from DRB1 molecules. Therefore, a decision was made to expand patient recruitment but focusing solely on DQ molecules.

Part 3: Expansion of data set, recruitment of patients from other centres and development and testing of a machine learning algorithm (units 1, 2 and 3). 
A total of 606 DNA samples, from 397 males and 209 female patients, were successfully typed. This included 320 patients from the United Kingdom, 259 from the United States and 27 from Australia. Patient demographics and clinical parameters can be seen in extended data tables 3 and 4 .

The clinical details of the training and validation sets are shown in extended data table 5. Table 1 shows the results of performance evaluation of the presented models on the test set. Taperdominated wearing THRs were excluded from the test set for the ALVAL pre-operative model to better fit the clinical context which this model would be exposed to (no MoM THRs are currently implanted in significant numbers - only resurfacings). For all models, the c-index and $\mathrm{ROC}(\mathrm{t})$ scores suggested a high degree of discrimination, whilst the IBS indicated good calibration and further backed up the indication of high discriminatory ability. The $\mathrm{ICl}$ scores supported the indication of good calibration and showed that at ten years, the weighted mean survival probability error was 1.8\% and $3.1 \%$ for pre-operative and post-operative ALVAL models respectively (extended data table 6). Extended data figures 1 and 2 show ALVAL ROC(t) for pre-operative and post-operative models from two to ten years after implantation. The ALVAL pre-operative model peaked in performance at two years after which a similar performance was observed from three to ten years. Similarly consistent performances were observed for the ALVAL post-operative model.

Figure 4. Kaplan Meier survival analysis for all female patients in the study (n=209), failure defined as revision secondary to ALVAL. The patients have been sub-grouped according to their HLA genotypes and Co concentration. Higher risk genotype defined by the top 50\% NTS binders, lower risk by the lowest $50 \%$. Cox proportional hazards modelling using "Co $<2$, lower risk genotype" as the reference group returned hazard ratios $(\mathrm{HR})(95 \% \mathrm{Cl})$ of $1.95(0.22-17.0)$ for $\mathrm{Co}<2$, higher risk genotype; 5.95 (0.62-57.2) for Co 2-4, lower risk genotype; 12.54 (1.62-97.2) for Co 2-4, higher risk genotype; 18.7 (2.45-142) for Co>4, lower risk genotype; 53.1 (7.26-388) for Co>4, higher risk genotype. 


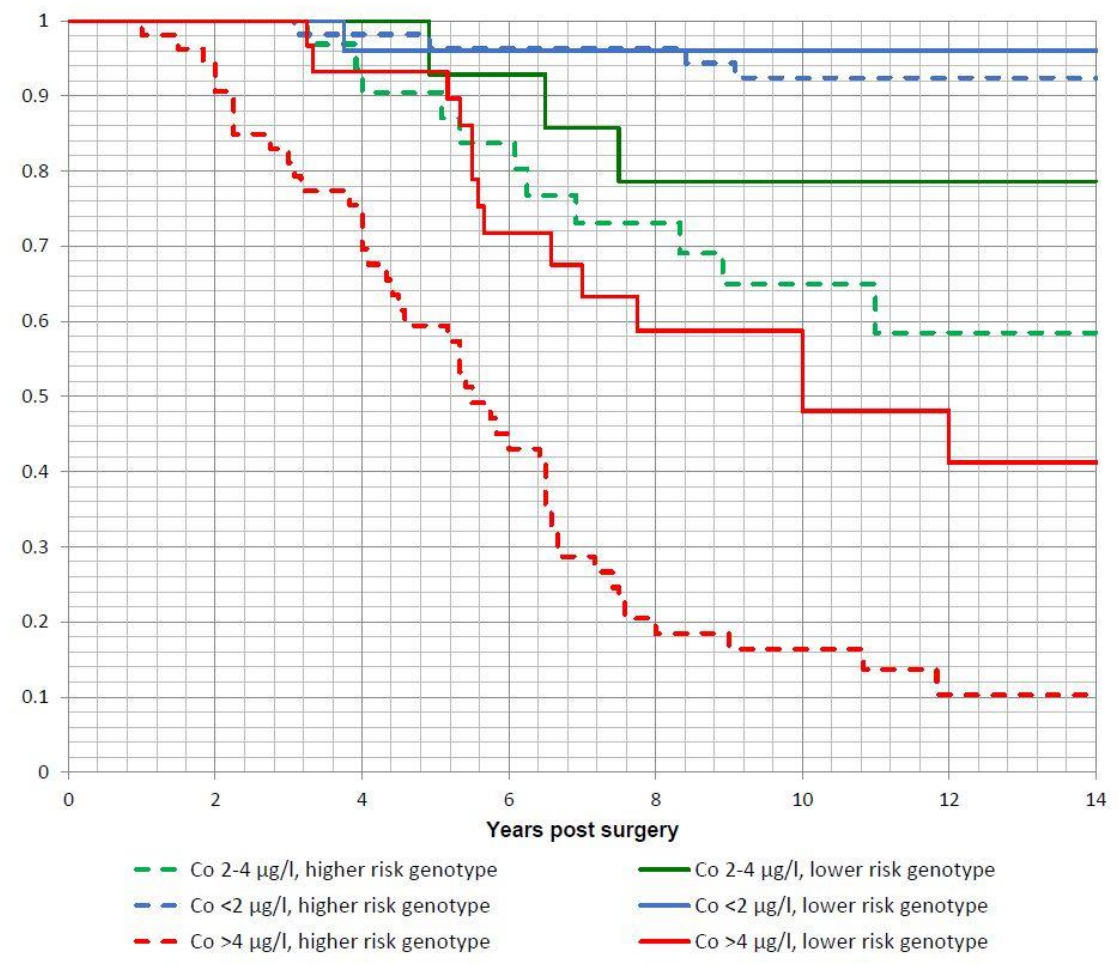

\section{Discussion}

At the height of its popularity around 15 years ago, MoM technology was used in around $30 \%$ of all hip replacements implanted in the United States[29]. In total, over 1 million MoM hips have been implanted across the world. Due to high complication rates, the use of MoM bearings has dramatically declined, and is now restricted to hip resurfacing procedures carried out in a limited number of centres.[3]

Research carried out over the last three decades - in the fields of dermatology and respiratory medicine - has identified HLA genes as key factors in the development of metal sensitivity. Orthopaedic researchers have also identified links between HLA genes and adverse local tissue responses, but, to date, the published studies have involved limited numbers of patients.[30, 31] Our results indicate that the development of DTH/ALVAL following joint replacement is determined by an interaction between patient sex, genotype and the volume of metal debris generated from a prosthesis. In present day clinical practise, genetic predisposition to DTH is not routinely considered 
nor tested for in the selection of an orthopaedic implant. Some centres carry out investigations such as skin patch testing or perform lymphocyte proliferation assays to identify patients who report a "metal allergy" preoperatively. However, these tests have faced continued scrutiny as to their accuracy and true clinical relevance.[32, 33] In this paper we have described the development and validation of an algorithm which may help identify patients at greater risk of DTH in order to guide implant selection and inform post-operative surveillance .

\section{Development of ALVAL is associated with HLA genotype}

In the initial part of this investigation, we demonstrated that patients developing ALVAL to low wearing prostheses possess different frequencies of specific HLA-DQ haplotypes when compared to those who remain asymptomatic at long term follow up. HLA-DQ haplotypes encoding for peptide binding grooves with greater affinity for the NTS of albumin present a higher risk of ALVAL. Albumin is the primary carrier for Co in both synovial fluid and serum. It has four metal ion binding sites, including the NTS. While the NTS is recognised to bind Co, under normal circumstances it is generally occupied by nickel or copper. However, this site can also bind Co ions, particularly when there are changes in the relative concentrations of metal in the surrounding fluid. The $\mathrm{N}$ terminal sequence also contains another site with even greater affinity for $\mathrm{Co}-$ site B, partially composed of His9 and Asp13. Metal ions can form complexes with self-proteins held at the peptide binding groove of APCs; these metal peptide complexes can provoke a response from $\mathrm{T} 4$ helper cells.

\section{Although patients display varying susceptibility, most patients require exposure to elevated wear rates to develop ALVAL.}

The presentation of a peptide:MHC complex by an APC does not automatically lead to sensitisation. This is demonstrated by the low ALVAL rates in our patients who possess "higher risk" haplotypes but have low blood metal ion concentrations. Sensitisation requires lymphocyte activation, and for a lymphocyte to become activated, the APC itself must be in an activated form. APCs possess innate PRRs (figure 2), which, when stimulated, promote activation and migration of APCs from the site of 
exposure to the draining lymph nodes. This process leads to expansion and survival of metal-reactive memory $T$ cells that circulate throughout the body. Metals can activate PRRs either directly, or indirectly, through the release of reactive oxygen species, the inflammasome pathway[34, 35] or via the induction of necrosis and release of alarmins.[36] An elevation in local metal ion concentrations, therefore, can not only raise the probability of metal:peptide neoantigen presentation, it can also increase the probability of APC activation and thus T cell sensitisation. Furthermore, an increase in the rate of implant wear can lead to the generation of larger particles which may frustrate effective macrophage phagocytosis, resulting in cell damage, the release of lysosomal products and a local reduction in $\mathrm{pH}$ levels.[37]

\section{The $\mathbf{N}$ terminal peptide sequence of albumin is recognised to fragment early in the endolysosomal} processing pathway.

Albumin peptides, as with other endogenous peptides, are constantly recycled in the body.[38] This recycling commences following pinocytosis or receptor-mediated cellular uptake, when proteins enter the endolysosomal pathway, and are exposed to an increasingly acidic environment (figure 2). As the $\mathrm{pH}$ drops, peptidases section the ingested proteins into their smaller constituent peptides. Albumin is protected from this endosomal degradation by the binding of neonatal Fc receptor (FcRn), binding which is initiated at $\mathrm{pH}$ values below 6.5.[39] However, albumin sequences 1-24 and 1-26 are some of the first to fragment under mildly acidic conditions,[40] a phenomenon which enables them to act as biomarkers in conditions such as graft versus host disease. Therefore, the NTS could detach from albumin via two mechanisms in different locations: in the synovial fluid itself, or in the endolysosomal pathway, where it is a front runner in the competition to bind with MHC II molecules. Once presented at the cellular membrane, NTS peptides would be exposed to metal ions released from the prosthesis, leading to the formation of metal:peptide complexes (figure 2).

Females are more susceptible to DTH. As is the case with other (largely HLA mediated) autoimmune/autoimmune like diseases, females develop ALVAL more readily than male patients. 
We have previously - incorrectly - ascribed this to the tendency for prostheses implanted into females to wear at higher rates. While it is indeed true that MoM hips implanted into females do tend to generate more wear debris, females appear to be more susceptible to ALVAL following exposure to equivalent amounts of metal debris. Accordingly, only females with low wearing prostheses, or genotypes associated with the lowest NTS binding affinity values, were associated with low rates of ALVAL. This finding was consistent with the literature.

Clinical implications. The results have implications for other types of joint replacements. Almost all commonly used total knee replacements (TKRs) incorporate at least one CoCr component. Yet, while it is now established that $\mathrm{CoCr}$ debris released from MoM hips is of great concern - necessitating specific guidance from orthopaedic societies[18] - there is a lack of consensus as to the clinical significance of metal sensitivity in the field of knee surgery. This may be due to a lack of standardisation in terminology, with the ill-defined condition "allergy" frequently referred to in the literature concerning knee prostheses.[41] It may be due to the pervasive belief that the amount of $\mathrm{CoCr}$ released from TKRs is negligible in comparison to that generated from MoM hips. However, this belief lies contrary to the findings reported in simulator and retrieval studies. $[42,43]$ In terms of clinical data, blood metal ion studies involving patients with TKRs are few in comparison to those on patients with MoM hips. The published studies report median Co concentrations ranging from between $0.28 \mu \mathrm{g} / \mathrm{l}$ (in patients with TKRs with titanium tibial trays)[44], to 4.28 in patients with bilateral knees with CoCr trays,[45] and up to $8.80 \mu \mathrm{g} / \mathrm{l}$ in patients with unstable components.[46] These ranges extend well beyond the levels which are associated with DTH/ALVAL in MoM hip patients who possess higher risk genotypes. Only recently have researchers performed large, targeted studies focusing on DTH/ALVAL in failed TKRs. Kurmis et al found a prevalence of pseudotumours or high-grade ALVALs in 7\% of their patients with failed TKAs.[47] These findings were substantiated by Crawford et al[48], who found that $19.1 \%$ of their patients who had undergone aseptic revision were found to have "perivascular lymphocytic infiltrate" on histological analysis. The aforementioned studies also identified a link between the extent of lymphocyte 
infiltration on the tissue specimens and the pain levels reported by the patients prior to the revision surgery, raising the possibility that DTH may be an under recognised cause of sub-optimal clinical outcomes following joint replacement. It is notable that studies consistently report complaints of chronic pain in approximately $20 \%$ of patients following TKA[49]. Our results indicate that at least $10 \%$ of individuals of European descent possess HLA genes which may respond unfavourably to relatively low levels of CoCr exposure.

\section{References for main text}

1. Pabinger, C. and A. Geissler, Utilization rates of hip arthroplasty in OECD countries. Osteoarthritis and Cartilage, 2014. 22(6): p. 734-741.

2. Willert, H.G., H. Bertram, and G.H. Buchhorn, Osteolysis in alloarthroplasty of the hip. The role of ultra-high molecular weight polyethylene wear particles. Clin Orthop Relat Res, 1990(258): p. 95-107.

3. 12th Annual Report. National Joint Registry of England and Wales, 2015.

4. Willert, H.G., et al., Metal-on-metal bearings and hypersensitivity in patients with artificial hip joints. A clinical and histomorphological study. J Bone Joint Surg Am, 2005. 87.

5. Langton, D., et al., Adverse reaction to metal debris following hip resurfacing: the influence of component type, orientation and volumetric wear. The Journal of bone and joint surgery. British volume, 2011. 93(2): p. 164-171.

6. Wawrzynski, J., et al., Hypersensitivity to Orthopedic Implants: A Review of the Literature. Rheumatology and Therapy, 2017. 4(1): p. 45-56.

7. Harris, W.H., The problem is osteolysis. Clin Orthop Relat Res, 1995(311): p. 46-53.

8. Treacy, R.B., C.W. McBryde, and P.B. Pynsent, Birmingham hip resurfacing arthroplasty. A minimum follow-up of five years. J Bone Joint Surg Br, 2005. 87(2): p. 167-70. 
9. Heisel, C., et al., Ten different hip resurfacing systems: biomechanical analysis of design and material properties. Int Orthop, 2009. 33(4): p. 939-43.

10. Pandit, H., et al., Pseudotumours associated with metal-on-metal hip resurfacings. J Bone Joint Surg Br, 2008. 90(7): p. 847-51.

11. Natu, S., et al., Adverse reactions to metal debris: histopathological features of periprosthetic soft tissue reactions seen in association with failed metal on metal hip arthroplasties. Journal of clinical pathology, 2012. 65(5): p. 409-418.

12. Nawabi, D.H., et al., MRI predicts ALVAL and tissue damage in metal-on-metal hip arthroplasty. Clinical orthopaedics and related research, 2014. 472(2): p. 471-481.

13. G., G., et al., Hip resurfacings revised for inflammatory pseudotumour have a poor outcome. The Journal of Bone and Joint Surgery. British volume, 2009. 91-B(8): p. 1019-1024.

14. Jameson, S.S., et al., The influence of age and sex on early clinical results after hip resurfacing: an independent center analysis. J Arthroplasty, 2008. 23(6 Suppl 1): p. 50-5.

15. Sidaginamale, R.P., et al., Blood metal ion testing is an effectivescreening tool to identify poorly performing metal-on-metal bearingsurfaces. Bone Joint Res, 2013. 2(5): p. 84-95.

16. De Smet, K., et al., Metal ion measurement as a diagnostic tool to identify problems with metal-on-metal hip resurfacing. J Bone Joint Surg Am, 2008. 90 Suppl 4: p. 202-8.

17. Hart, A.J., et al., The painful metal-on-metal hip resurfacing. J Bone Joint Surg Br, 2009. 91(6): p. 738-44.

18. http://www.mhra.gov.uk/home/groups/dts bs/documents/medicaldevicealert/con155767.pdf.

19. Liow, M.H., et al., Metal Ion Levels Are Not Correlated With Histopathology of Adverse Local Tissue Reactions in Taper Corrosion of Total Hip Arthroplasty. J Arthroplasty, 2016. 31(8): p. 1797-802. 
20. Langton, D.J., et al., The clinical implications of elevated blood metal ion concentrations in asymptomatic patients with MoM hip resurfacings: a cohort study. BMJ open, 2013. 3(3): p. e001541.

21. Wysocki, T., M. Olesińska, and A. Paradowska-Gorycka, Current Understanding of an Emerging Role of HLA-DRB1 Gene in Rheumatoid Arthritis-From Research to Clinical Practice. Cells, 2020. 9(5).

22. Sollid, L.M., The roles of MHC class II genes and post-translational modification in celiac disease. Immunogenetics, 2017. 69(8-9): p. 605-616.

23. Richeldi, L., R. Sorrentino, and C. Saltini, HLA-DPB1 glutamate 69: a genetic marker of beryllium disease. Science, 1993. 262(5131): p. 242-4.

24. Lison, D., et al., Experimental research into the pathogenesis of cobalt/hard metal lung disease. Eur Respir J, 1996. 9(5): p. 1024-8.

25. Büdinger, L. and M. Hertl, Immunologic mechanisms in hypersensitivity reactions to metal ions: an overview. Allergy, 2000. 55(2): p. 108-15.

26. Sinigaglia, F., The molecular basis of metal recognition by T cells. J Invest Dermatol, 1994. 102(4): p. 398-401.

27. Rosenman, K.D., et al., HLA class II DPB1 and DRB1 polymorphisms associated with genetic susceptibility to beryllium toxicity. Occup Environ Med, 2011. 68(7): p. 487-93.

28. Griem, P., et al., $T$ cell cross-reactivity to heavy metals: identical cryptic peptides may be presented from protein exposed to different metals. Eur J Immunol, 1998. 28(6): p. 1941-7.

29. Bozic, K.J., et al., The epidemiology of bearing surface usage in total hip arthroplasty in the United States. J Bone Joint Surg Am, 2009. 91(7): p. 1614-20.

30. Kilb, B.K.J., et al., Frank Stinchfield Award: Identification of the At-risk Genotype for Development of Pseudotumors Around Metal-on-metal THAs. Clin Orthop Relat Res, 2018. 476(2): p. 230-241. 
31. Blowers, P., Immune system involvement in metal hip implant failure. 2015, University of East Anglia.

32. Yang, S., et al., Lymphocyte Transformation Testing (LTT) in Cases of Pain Following Total Knee Arthroplasty: Little Relationship to Histopathologic Findings and Revision Outcomes. JBJS, 2019. 101(3): p. 257-264.

33. Haddad, S.F., et al., Exploring the Incidence, Implications, and Relevance of Metal Allergy to Orthopaedic Surgeons. J Am Acad Orthop Surg Glob Res Rev, 2019. 3(4): p. e023.

34. Caicedo, M.S., et al., Increasing both CoCrMo-alloy particle size and surface irregularity induces increased macrophage inflammasome activation in vitro potentially through lysosomal destabilization mechanisms. J Orthop Res, 2013. 31(10): p. 1633-42.

35. Yazdi, A.S., K. Ghoreschi, and M. Röcken, Inflammasome Activation in Delayed-Type Hypersensitivity Reactions. Journal of Investigative Dermatology, 2007. 127(8): p. 1853-1855.

36. McKee, A.S., et al., MyD88 dependence of beryllium-induced dendritic cell trafficking and CD4+ ${ }^{+}$-cell priming. Mucosal Immunol, 2015. 8(6): p. 1237-47.

37. Perino, G., et al., The contribution of the histopathological examination to the diagnosis of adverse local tissue reactions in arthroplasty. EFORT Open Reviews, 2021. 6(6): p. 399-419.

38. Peters, T., 6 - Clinical Aspects: Albumin in Medicine, in All About Albumin, T. Peters, Editor. 1995, Academic Press: San Diego. p. 251-284.

39. Chaudhury, C., et al., The major histocompatibility complex-related Fc receptor for IgG (FcRn) binds albumin and prolongs its lifespan. Journal of Experimental Medicine, 2003. 197(3): p. 315-322.

40. Yang, J., et al., Mass spectrometric characterization of limited proteolysis activity in human plasma samples under mild acidic conditions. Methods, 2015. 89: p. 30-7.

41. Saccomanno, M.F., et al., Allergy in total knee replacement surgery: Is it a real problem? World J Orthop, 2019. 10(2): p. 63-70. 
42. Kretzer, J.P., et al., Wear in total knee arthroplasty--just a question of polyethylene?: Metal ion release in total knee arthroplasty. Int Orthop, 2014. 38(2): p. 335-40.

43. Arnholt, C.M., et al., Corrosion Damage and Wear Mechanisms in Long-Term Retrieved CoCr Femoral Components for Total Knee Arthroplasty. J Arthroplasty, 2016. 31(12): p. 2900-2906.

44. Reiner, T., et al., Blood Metal Ion Release After Primary Total Knee Arthroplasty: A Prospective Study. Orthop Surg, 2020. 12(2): p. 396-403.

45. Luetzner, J., et al., Serum metal ion exposure after total knee arthroplasty. Clin Orthop Relat Res, 2007. 461: p. 136-42.

46. Savarino, L., et al., The potential role of metal ion release as a marker of loosening in patients with total knee replacement: a cohort study. J Bone Joint Surg Br, 2010. 92(5): p. 634-8.

47. Kurmis, A.P., et al., Pseudotumors and High-Grade Aseptic Lymphocyte-Dominated VasculitisAssociated Lesions Around Total Knee Replacements Identified at Aseptic Revision Surgery: Findings of a Large-Scale Histologic Review. J Arthroplasty, 2019. 34(10): p. 2434-2438.

48. Crawford, D.A., et al., Impact of perivascular lymphocytic infiltration in aseptic total knee revision. Bone Joint J, 2021. 103-b(6 Supple A): p. 145-149.

49. Wylde, V., et al., Chronic pain after total knee arthroplasty. EFORT open reviews, 2018. 3(8): p. 461-470. 


\section{Methods: Patients and hospital centres}

The study commenced at a single centre (centre 1, United Kingdom) where a large number of metalon-metal hip arthroplasties were performed between 2002 and 2010. These patient cohorts have been described in full in previous publications.[5] The patients have been kept under surveillance with annual clinical review and blood metal ion testing. As part of an ethically approved project (commenced in 2008, IRAS reference 14119), patients who undergo revision of their MoM hip prostheses have: undergone metal ion testing to assess $\mathrm{Co}$ and $\mathrm{Cr}$ concentrations in their blood, serum and hip joint synovial fluid samples; their explanted prostheses analysed to determine their volumetric wear; tissue samples excised at revision surgery assessed by a specialist histopathologist (SN). The total number of revision cases in the database at commencement of study was 420 .

\section{Blood/serum metal ion testing}

We have carried out a substantial amount of work detailing the relationships between volumetric wear of implants and the corresponding concentrations of $\mathrm{Co}$ and $\mathrm{Cr}$ ion in the blood, serum and synovial fluid fractions.[50] Samples were tested using validated methodology.[51, 52]

Wear analysis. Explanted prostheses were analysed using a coordinate measuring machine (Legex 322; Mitutoyo Ltd, Halifax, United Kingdom) to calculate the total amount of material that had been removed from the components in vivo. This material loss can be expressed in volumetric terms as 'total volumetric wear' (in $\mathrm{mm}^{3}$ ) or the total value can be divided by the number of years in vivo to provide a mean 'volumetric wear rate' (expressed in $\mathrm{mm}^{3} / \mathrm{year}$ ). The accuracy of such methods has been validated and is of the order of $0.5 \mathrm{~mm}^{3}$ per component for bearings and $0.2 \mathrm{~mm}^{3}$ for tapers.[53] Throughout this paper, wear rates refer only to volumetric Co-Cr material loss. For resurfacings, 'total volumetric wear rates' refer to the bearing surface wear rates (combined head and acetabular component volumetric wear rates). For THAs, 'total volumetric wear rates' refer to the combined wear rates of the bearings and female taper surface. THAs in the study were used with 
titanium stems. We have previously confirmed that titanium release is small in comparison with CoCr.[54]

Histopathological tissue assessment. This was carried out as has previously been described in greater detail. Samples were taken from between two and four sites surrounding the implant. Up to ten paraffin blocks were processed per site to ensure the viable tissue was well represented. Samples were also sent to the microbiology department to identify sepsis. A single consultant histopathologist (SN), examined the slides independently of the clinical findings, blinded to the results of the wear or fluid metal ion analyses. Note: Adverse reaction to metal debris (ARMD) is an umbrella term which refers to clinical signs and symptoms occurring in association with metal debris.[55] The typical response to metal debris is limited to a macrophage infiltrate.[56] ALVAL is a subset of ARMD, referring to the additional lymphocyte infiltrate and histological features of DTH.

The hallmark of ALVAL is the development of a perivascular lymphocytic infiltrate which appears to be dynamic process. Thin perivascular cuffs initially appear which increase in thickness as the recruitment of lymphocytes is further stimulated. These cuffs then either expand to develop into aggregates or coalesce into one another, forming larger aggregates. Once this process of lymphoid neogenesis is complete, pale germinal centres appear. The ALVAL response was graded from 0 (absent) to 3 (severe) according to the integrity of the synovial membrane and the stage of lymphocytic infiltration. This classification system has shown good intra and interobserver reliability.[57]

\section{Part 1: Investigation of genetic associations using extreme phenotype group comparison}

From the hospital database, we identified four groups of patients, to represent the different phenotypes:

1. Patients with joint failure who developed moderate/severe ALVAL in association with prostheses wearing at lower than the median wear rate of the total revision cohort. 
2. Patients with joint failure who developed moderate/severe ALVAL in association with prostheses wearing at greater than the median wear rate of the total revision cohort.

3. Patients with joint failure with a pathological response limited to macrophage infiltration; no lymphocyte infiltration identified.

4. Patients with joints remaining in situ. Patients who were pain free and satisfied with the results of their hip arthroplasties at a minimum of ten years post surgery.

We wrote to these patients explaining the nature of the study and invited them to submit a sample for DNA analysis.

DNA sample collection and processing

A combination of ORAcollect OCR-100 buccal swabs and Oragene DNA OG-610 saliva collection kits (both DNA Genotek Inc, Ontario, Canada) were used to collect samples for DNA extraction. DNA was extracted from the swab and saliva samples using the Roche MagnaPure Compact automated platform (Roche Holding AG, Switzerland). DNA was then quantified using the Thermo Fisher Qubit dsDNA BR Assay kit and standardised to $25 \mathrm{ng} / \mu \mathrm{l}$. HLA genotyping was performed using the One Lambda AllType NGS kits (One Lambda, USA), with the Illumina MiSeq platform (Illumina, USA). Full gene sequencing was carried out for HLA-A, -B, -C, -DQA1 and -DPA1 and partial gene sequencing (omission of exon 1) for HLA-DRB1, -DRB345, -DQB1 and -DPB1. HLA genotypes were analysed using One Lambda TypeStream Visual 1.3 software (One Lambda, USA).

Global locus-wise association for each HLA gene was performed using UNPHASED v 3.0.13. Haplotypes were estimated for DRB1-DQA1-DQB1 also in UNPHASED.[58] The distribution of the HLA class I and II alleles were compared between groups using a standard approach as previously described.[59] 


\section{Part 2: In silico analysis of peptide-HLA class II binding affinity and Cox proportional hazards}

modelling

Published literature indicates that ALVAL is associated with the accumulation of large, albumin rich synovial fluid collections.[57] Fluid taken from patients with ALVAL also tends to contain a disproportionate elevation of metal ions in the synovial fluid compared to that which would be expected from the rate of material loss from the implant $[57,60]$. This elevation is more pronounced with respect to Co ions in comparison to Cr. Furthermore, it is recognised that protein binding of metal ions delays their egression from the joint space, and that Co binds almost exclusively to albumin in synovial fluid and serum samples. $[61,62]$ Collectively, these observations imply that albumin bound Co is a key factor in the development of ALVAL.

\section{Candidate antigen}

The most likely candidate sequence we believed, would be the N-terminal binding site (NTS) which contains two metal ion binding sites. The first arises from the first triplet amino acid motif of albumin: Asp1-Ala2-His3.[63] The second (also termed site B), is partially composed of His9 and Asp13. The $\mathrm{N}$ terminal sequence is the most likely to be sectioned early in the cellular processing of albumin following its uptake by APCs. While site B exhibits greater binding affinity for Co, the NTS binding site is formed from a singular domain, continuous amino acid sequence, not reliant on connections between domains to maintain its metal binding properties.[63]

\section{Peptide binding analysis}

Validated software enables virtual construction of peptide binding grooves encoded by an individual's HLA genotype.[64] This allows the calculation of the binding affinity between a particular HLA encoded peptide binding groove and an array of naturally occurring peptides. Using this software, in this investigation, we initially sought to: identify HLA genes associated with the development of ALVAL; determine whether HLA genes associated with the development of ALVAL at 
low rates of wear encode for peptide binding grooves with higher affinities for the $\mathrm{N}$ terminal metal binding sites of albumin.

We performed in silico analysis of peptide-HLA class II binding affinity across HLA proteins found in our patient population. All HLA-DQA1, -DQB1, and DRB1 alleles were selected to assess the peptidebinding affinity of their corresponding peptide binding proteins. HLA-DR is represented by HLADRA/DRB1 dimer. Since HLA-DRA is considered monomorphic, we only used HLA-DRB1. HLA-DQ is represented by the HLA-DQA1/DQB1 dimer.

FASTA-formatted protein sequence data were retrieved from the UniProt database (www.uniprot.org) for human serum albumin (P02768). We extracted the $\mathrm{N}$ terminal 15 amino acid sequence DAHKSEVAHRFKDLG, a sequence which includes two recognised Co binding sites. Predictions for HLA binding to this sequence were performed using NetMHCllpan4.0.[64] The rank binding affinities were calculated for all the possible DQ and DRB1 combinations. We used the \%EL rank score as the primary binding metric, as advised in personal communication with the software developers.[65] We investigated whether the binding scores influenced the risk of developing ALVAL over time using Cox proportional hazards modelling. Multiple survival models were constructed to explain the development of time dependent prosthetic failure associated with mild/moderate or severe ALVAL, using the following independent variables: NTS binding affinity; pre revision blood Co concentrations; pre revision blood $\mathrm{Cr}$ concentrations; patient sex; patient age at time of primary surgery; presence of bilateral prostheses; type of prosthesis (THR versus resurfacing arthroplasty).

\section{Part 3: Expansion of data set, inclusion of patients from other centres and development of machine learning algorithm}

We then invited all remaining patients in the database who had undergone revision surgery for whom there was a full complement of clinical data, including explanted components available for analysis. We also invited all remaining patients under regular follow up who were recorded to be asymptomatic at greater than ten years follow up. Concurrently, we expanded the study to include 
two other units. Centre 2 is an orthopaedic specialist unit in New York, United States. Centre 3 is a teaching hospital in Western Australia. The units manage the follow up of MoM patients in a similar way and also routinely carry out analysis of explanted components. A similar research protocol was followed, with patients who were asymptomatic as well as those who had experienced failure of their joints invited to give a sample for DNA analysis. The same parameters were recorded as at centre 1 . When all samples had been analysed, the data set was randomly split $70 / 30$, with the larger set used to train a machine learning algorithm for the prediction of the development of ALVAL. The remaining data was held back, blinded from the analysts and used to test the algorithm when it was finalised.

Two models were trained to predict hazard ratios and survival functions up to ten years after implantation of a MoM prosthesis for pre-operative and post-operative patients:

1. Preoperative prediction of development of ALVAL. For this model metal exposure was divided into two groups: low wear (Co concentrations stabilise to $<2 \mu \mathrm{g} / \mathrm{I}$ ) and increased wear (Co concentrations stabilise to $\geq 2$ and $\leq 4 \mu \mathrm{g} / \mathrm{l}$ ). $4 \mu \mathrm{g} / \mathrm{l}$ equates to approximately three times the wear rate of a well-functioning device. It was therefore not felt necessary to provide a preoperative prediction for metal concentrations above this level

2. Postoperative prediction of development of ALVAL, in which actual measured $\mathrm{Co}$ and $\mathrm{Cr}$ concentrations could be used in the modelling

\section{Statistics and machine learning approach}

To select a machine learning model which generalises well, one must disjoint the training and testing strategy. As the training and test set are assumed to be drawn from the same probability distribution, they should be identically distributed.[66] Therefore, we formulated our test set by randomly sampling the full dataset (without replacement) stratified on the event indicator. The training data comprised of the remaining samples. 
Feature engineering was carried out on the training data to identify features that best predicted risk of failure due to ARMD and ALVAL within ten years of implantation of a MoM prosthesis. Boruta[67], a random forest feature selection algorithm was applied to 2939 features, generated from a combination of: patient features; binding affinities of cis and trans haplotypes; binary presence of cis and trans haplotypes; cis and trans haplotype gene dosage; thresholding binding affinities of cis and trans haplotypes to generate categorical features; polynomial and interaction features. The algorithm removed features that were identified as being less relevant than random features in an iterative supervised fashion. This feature selection strategy avoids overfitting when assessing the statistical significance of many features. Features that were identified as being associated with ALVAL were used to train gradient boosted survival analysis machine learning models with a Cox proportional hazards loss function and a regression tree base learner. A gradient boosted survival analysis model iteratively fits regression trees to minimise the negative Cox log-partial likelihood.[68, 69] The Cox log-partial likelihood is at a maximum when the failure and censoring times are fully concordant. Therefore, a model that is trained to minimise the negative of this function (by iteratively fitting regression trees), will attempt to predict concordant results accounting for both failure times and censoring. Regularisation was employed to reduce overfitting on the training data. Nested 5-fold cross-validation (CV) was used on the training data to enable better estimation of generalisation error and reduce model selection bias. Nested CV is preferred over flat CV as it separates the tuning of the model from the estimation of the generalisation error, reducing overfitting on the model selection criterion. [70] Nested CV has shown to be unnecessary for most practical applications except for when the number of hyper-parameters is large[70, 71], as is the case for gradient boosted survival analysis models, hence its use in our validation strategy. Hyperparameters of both models were optimised using a successive halving random search, which optimises hyper-parameters faster than the traditionally used grid search method.[72, 73] Integrated Brier Score was chosen as the scoring function as our model's primary use was predicting 
hazard ratios and survival curves and therefore discriminatory ability and calibration were equally important. Cross-validated probability calibration did not yield improvements in IBS and ICI.

After selecting the best performing model based on the IBS assessed on the training data, the model was then used to predict on the test set. IBS, Uno's c-index, time-dependent AUROC (ROC(t)), and Integrated Calibration Index (ICI) performance statistics were then computed on the test set. Brier score is the mean squared error of the predicted survival probability at a given time point. IBS summarises the Brier score over a particular time interval, representing overall model performance. A low Brier score verifies a high degree of discrimination and calibration.[74] Harrell's c-index is a rank-correlation measure which is an estimate of the probability that for a randomly selected pair of samples, the patient with the lower risk score outlives the patient with the higher risk score.[75] We opted to use Uno's variation of c-index for measuring discriminatory performance, which addresses the overly-optimistic results observed for Harrell's c-index with increasing censoring frequency.[76] Whilst AUROC is equivalent to c-index for binary outcomes, $R O C(t)$ provides a measure of performance for a given time of interest. We used an $\mathrm{ROC}(\mathrm{t})$ measure which accounts for censored patients using the Kaplan-Meier estimator to assess discriminatory performance at discrete time periods.[77]

When a model is tasked with predicting probabilities such as those seen in survival curves, verification of good calibration is important such that predicted probabilities closely follow observed frequencies of survival - ICl may be used to quantify this. ICI is the mean absolute difference between observed and predicted probabilities, weighted by the probability density function of the distribution of predicted probabilities. [78] We used Austin et al.'s adaptation of ICI for survival analysis problems.[79] As the training and testing of the model was disjointed, the performance statistics evaluated on the test set provided measures of how well each model would generalise to unseen data. Confidence intervals were estimated using the Bootstrap method. After completion of performance evaluation, each model was refit on the training and test data and hyper-parameters 
were retuned. The models were then serialised and integrated into a cloud-hosted pipeline for inference via a web app.

\section{References for methodology}

5. Langton, D., et al., Adverse reaction to metal debris following hip resurfacing: the influence of component type, orientation and volumetric wear. The Journal of bone and joint surgery. British volume, 2011. 93(2): p. 164-171.

50. Sidaginamale, R., et al., Blood metal ion testing is an effective screening tool to identify poorly performing metal-on-metal bearing surfaces. Bone \& joint research, 2013. 2(5): p. 8495.

51. Langton, D.J., et al., The effect of component size and orientation on the concentrations of metal ions after resurfacing arthroplasty of the hip. J Bone Joint Surg Br, 2008. 90(9): p. 1143-51.

52. Langton, D., et al., Is the synovial fluid cobalt-to-chromium ratio related to the serum partitioning of metal debris following metal-on-metal hip arthroplasty? Bone \& joint research, 2019. 8(3): p. 146-155.

53. Langton, D., et al., Investigation of taper failure in a contemporary metal-on-metal hip arthroplasty system through examination of unused and explanted prostheses. JBJS, 2017. 99(5): p. 427-436.

54. Langton, D.J., et al., A comparison study of stem taper material loss at similar and mixed metal head-neck taper junctions. Bone Joint J, 2017. 99-b(10): p. 1304-1312.

55. Reito, A., et al., Prevalence of Failure due to Adverse Reaction to Metal Debris in Modern, Medium and Large Diameter Metal-on-Metal Hip Replacements - The Effect of Novel 
Screening Methods: Systematic Review and Metaregression Analysis. PLoS One, 2016. 11(3): p. e0147872.

56. Campbell, P., S.H. Park, and E. Ebramzadeh, Semi-quantitative histology confirms that the macrophage is the predominant cell type in metal-on-metal hip tissues. J Orthop Res, 2021.

57. Langton, D.J., et al., Aseptic lymphocyte-dominated vasculitis-associated lesions are related to changes in metal ion handling in the joint capsules of metal-on-metal hip arthroplasties. Bone Joint Res, 2018. 7(6): p. 388-396.

58. Dudbridge, F., Likelihood-based association analysis for nuclear families and unrelated subjects with missing genotype data. Hum Hered, 2008. 66(2): p. 87-98.

59. Langton, D.J., et al., The influence of HLA genotype on the severity of COVID-19 infection. Hla, 2021. 98(1): p. 14-22.

60. Houdek, M.T., et al., Synovial Fluid Metal Ion Levels are Superior to Blood Metal Ion Levels in Predicting an Adverse Local Tissue Reaction in Failed Total Hip Arthroplasty. The Journal of Arthroplasty, 2021.

61. Wallis, W.J., P.A. Simkin, and W.B. Nelp, Protein traffic in human synovial effusions. Arthritis Rheum, 1987. 30(1): p. 57-63.

62. Simkin, P.A. and J.E. Bassett, Pathways of microvascular permeability in the synovium of normal and diseased human knees. J Rheumatol, 2011. 38(12): p. 2635-42.

63. Lakusta, H. and B. Sarkar, Equilibrium studies of zinc(II) and cobalt(II) binding to tripeptide analogues of the amino terminus of human serum albumin. Journal of Inorganic Biochemistry, 1979. 11(4): p. 303-315.

64. Jensen, K.K., et al., Improved methods for predicting peptide binding affinity to MHC class II molecules. Immunology, 2018. 154(3): p. 394-406.

65. Reynisson, B., et al., Improved Prediction of MHC II Antigen Presentation through Integration and Motif Deconvolution of Mass Spectrometry MHC Eluted Ligand Data. J Proteome Res, 2020. 19(6): p. 2304-2315. 
66. Goodfellow, I., Y. Bengio, and A. Courville, Deep learning. 2016: MIT press.

67. Kursa, M.B. and W.R. Rudnicki, Feature Selection with the Boruta Package. 2010, 2010. 36(11): p. 13.

68. Ridgeway, G. The State of Boosting 1999.

69. COX, D.R., Partial likelihood. Biometrika, 1975. 62(2): p. 269-276.

70. Cawley, G.C. and N.L.C. Talbot, On Over-fitting in Model Selection and Subsequent Selection Bias in Performance Evaluation. J. Mach. Learn. Res., 2010. 11: p. 2079-2107.

71. Wainer, J. and G. Cawley, Nested cross-validation when selecting classifiers is overzealous for most practical applications. Expert Systems with Applications, 2021. 182: p. 115222.

72. Jamieson, K. and A. Talwalkar. Non-stochastic best arm identification and hyperparameter optimization. in Artificial Intelligence and Statistics. 2016. PMLR.

73. Li, L., et al., Hyperband: A novel bandit-based approach to hyperparameter optimization. The Journal of Machine Learning Research, 2017. 18(1): p. 6765-6816.

74. Harrell, F.E., Missing data, in Regression modeling strategies. 2001, Springer. p. 41-52.

75. Harrell, F.E., et al., Evaluating the yield of medical tests. Jama, 1982. 247(18): p. 2543-2546.

76. Uno, $\mathrm{H}$., et al., On the C-statistics for evaluating overall adequacy of risk prediction procedures with censored survival data. Statistics in medicine, 2011. 30(10): p. 1105-1117.

77. Heagerty, P.J. and P. Saha, SurvivalROC: time-dependent ROC curve estimation from censored survival data. Biometrics, 2000. 56(2): p. 337-344.

78. Austin, P.C. and E.W. Steyerberg, The Integrated Calibration Index (ICI) and related metrics for quantifying the calibration of logistic regression models. Statistics in medicine, 2019. 38(21): p. 4051-4065.

79. Peter, C.A., E.H. Frank Jr, and v.K. David, Graphical Calibration Curves and the Integrated Calibration Index (ICI) for Survival Models. Statistics in medicine. 


\section{Author contributions}

David J Langton: Devised concept of the study, study design, coordination, first draft and approval of manuscript.

Rohan M Bhalekar: Sample collection, explant analysis, data collection, review and approval of manuscript.

Thomas J Joyce: Initiated original study databases, explant analysis, drafting and approval of manuscript.

Stephen P Rushton: Review of statistical methodology and data review. Drafting and approval of manuscript.

Benjamin J Wainwright: Study design, first draft and approval of manuscript.

Matthew E Nargol: Statistical analysis and machine learning algorithm development, review and approval of manuscript.

Nish Shyam: Statistical analysis and machine learning algorithm development, review and approval of manuscript.

Benedicte Lie: Statistical analysis (genetics), drafting and approval of manuscript.

Moreica Pabbruwe: Explant analysis and coordination of study at Australian centre, drafting and approval of manuscript.

Alan Stewart: Guidance on methodology with respect to peptide modelling and metalloprotein binding. Drafting and approval of final manuscript.

Susan Waller: Data collection, maintenance of study database at centre 1, patient review, approval of manuscript. 
Shonali Natu: Initiated original study database, analysis of samples, drafting and approval of manuscript.

Renne Ren: Data collection, maintenance of study database in United States, patient review, approval of manuscript.

Rachelle Hornick: Data collection, maintenance of study database in United States, patient review, approval of manuscript.

Rebecca Darlay: Statistical analysis (genetics), drafting and approval of manuscript.

Edwin Su: Senior surgeon at United States centre, involved in study design, drafting and approval of manuscript.

Antoni VF Nargol: Senior orthopaedic surgeon at centre 1, initiated original study database, patient review, data interpretation, drafting and approval of manuscript.

Conflicts of interest: The algorithm described in this study has been developed into a piece of software to be used as a medical device ("Orthotype"). Orthotype has been patented, and is owned by the company PXD Ltd, trading as ExplantLab. David Langton, the lead author is director of this company.

Data availability statement: The data that support the findings of this study are available from the corresponding author upon reasonable request.

Code availability statement: The computer code software algorithm is proprietary and therefore not available to the general reader. The code will be made available to the reviewers during the peer review process. 


\section{Extended data}

Extended data table 1. Extreme phenotype analysis. All class I and class II HLA alleles were typed to six digit resolution. However, only four digit values were used in the comparisons between groups and in the modelling. The full set of allele frequency comparisons is shown in supplementary data table 1.

\begin{tabular}{|c|c|c|c|c|c|}
\hline & $\begin{array}{l}\text { Revised: ALVAL, } \\
\text { low wear (27) }\end{array}$ & $\begin{array}{c}\text { Revised: } \\
\text { Macrophage } \\
\text { only (37) }\end{array}$ & $\begin{array}{l}\text { Low wear ALVAL } \\
\text { vs macrophage } \\
\text { only p value }\end{array}$ & $\begin{array}{l}\text { Asymptomatic } \\
>10 \text { years (61) }\end{array}$ & $\begin{array}{c}\text { ALVAL vs } \\
\text { asymptomatic } P \\
\text { value }\end{array}$ \\
\hline Male : female & $6: 21$ & 20:37 & 0.010 & 45:16 & $<0.001$ \\
\hline Median (range) age & $65(42-76)$ & $58(29-70)$ & 0.010 & $56(34-75)$ & $<0.001$ \\
\hline Resurfacings vs THRs & $2: 25$ & 22:37 & 0.003 & 61:0 & $<0.001$ \\
\hline $\begin{array}{l}\text { DQA1*01:01- } \\
\text { DQB1*05:01 }\end{array}$ & $3 / 54(5.5 \%)$ & $12 / 74(15.8 \%)$ & 0.087 & 21/122 (17.2\%) & 0.026 \\
\hline $\begin{array}{l}\text { DQA1*01:02- } \\
\text { DQB1*06:02 }\end{array}$ & 9/54 (16.7\%) & $15 / 74(20.2 \%)$ & 0.772 & 23/122 (18.4\%) & 0.891 \\
\hline $\begin{array}{l}\text { DQA1*02:01- } \\
\text { DQB1*02:02 }\end{array}$ & $11 / 54(20.3 \%)$ & 10/74 (13.5\%) & 0.402 & $6 / 122(4.9 \%)$ & 0.015 \\
\hline $\begin{array}{l}\text { DQA1*03:01- } \\
\text { DQB1*03:02 }\end{array}$ & 3/54 (5.6\%) & $7 / 74(9.5 \%)$ & 0.404 & 9/122 (7.4\%) & 0.651 \\
\hline $\begin{array}{l}\text { DQA1*05:01- } \\
\text { DQB1*02:01 }\end{array}$ & 9/54 (16.7\%) & $12 / 74(16.2 \%)$ & 1.000 & 23/122 (12.3\%) & 0.606 \\
\hline $\begin{array}{l}\text { DQA1*05:05- } \\
\text { DQB1*03:01 }\end{array}$ & 10/54 (18.5\%) & 3/74 (4.1\%) & 0.026 & 7/122 (5.7\%) & 0.044 \\
\hline
\end{tabular}


Extended data table 2. Cox proportional hazards modelling from phase 1: Centre 1 patients only, $\mathrm{N}=$ 161.

\begin{tabular}{|c|c|c|c|c|c|c|}
\hline \multicolumn{7}{|c|}{ Model 1: Survival based on ALVAL severity of mild, moderate or severe } \\
\hline Variable & Coeff & $\begin{array}{c}\text { Standard } \\
\text { error }\end{array}$ & $P$ value & $\begin{array}{l}\text { Hazard } \\
\text { ratio } \\
\text { (HR) }\end{array}$ & $\begin{array}{c}\text { HR } \\
\text { Lower Cl } \\
(95 \%)\end{array}$ & $\begin{array}{c}\text { HR } \\
\text { Upper C } \\
(95 \%)\end{array}$ \\
\hline $\begin{array}{l}\text { Log normalised cobalt } \\
\text { concentration }\end{array}$ & 1.575 & 0.153 & $<0.001$ & 4.829 & 3.577 & 6.518 \\
\hline Age & 0.015 & 0.014 & 0.308 & 1.015 & 0.987 & 1.044 \\
\hline Rank binding affinity for NTS & -1.062 & 0.435 & 0.015 & 0.346 & 0.147 & 0.812 \\
\hline Sex-M & -0.430 & 0.216 & 0.047 & 0.651 & 0.426 & 0.994 \\
\hline Type-THR & 0.414 & 0.231 & 0.074 & 1.512 & 0.961 & 2.380 \\
\hline \multicolumn{7}{|c|}{ Model 2: Survival based on ALVAL severity of moderate or severe } \\
\hline Rank binding affinity for NTS & -2.161 & 0.573 & $<0.001$ & 0.115 & 0.038 & 0.354 \\
\hline $\begin{array}{l}\text { Log normalised cobalt } \\
\text { concentration }\end{array}$ & 1.598 & 0.200 & $<0.001$ & 4.941 & 3.341 & 7.307 \\
\hline Age & 0.033 & 0.019 & 0.083 & 1.034 & 0.996 & 1.074 \\
\hline Sex-M & -0.370 & 0.277 & 0.182 & 0.691 & 0.402 & 1.189 \\
\hline Type-THR & 0.455 & 0.307 & 0.139 & 1.576 & 0.863 & 2.879 \\
\hline
\end{tabular}


Extended data table 3. Clinical details of all the patients from all centres in the study, divided by clinical outcome.

\begin{tabular}{|l|c|c|c|}
\hline & Total & Asymptomatic & Failed \\
\hline Total number of patients & 606 & 430 & 176 \\
\hline Total number of hips & 711 & 535 & 176 \\
\hline Follow up (years) & $10(1-20)$ & $12(3-20)$ & $6(1-15)$ \\
\hline Age (range) & $55(25-85)$ & $54(25-78)$ & $58(25-85)$ \\
\hline \% male patients & $66 \%(397: 209)$ & $74 \%(320: 110)$ & $44 \%(77: 99)$ \\
\hline Resurfacings vs THRs & 468 vs $138(77 \%)$ & 43 vs $430(90 \%)$ & $46 \%(81: 75)$ \\
\hline$\%$ patients with bilateral & $24 \%$ & $24 \%$ & $24 \%$ \\
\hline BMI & 26.6 & 26.7 & $7.6(0.7-271.0)$ \\
\hline Median (range) Co $(\mu \mathrm{g} / \mathrm{l})$ & $2.0(0.1-271.0)$ & $1.5(0.1-34.4)$ & $7.0(0.7-108.4)$ \\
\hline Median (range) Cr $(\mu \mathrm{g} / \mathrm{l})$ & $2.5(0.2-108.4)$ & $2(0.2-18.6)$ & \\
\hline
\end{tabular}


Extended data table 4. Clinical details of patients who underwent revision of their prostheses.

\begin{tabular}{|c|c|c|c|c|}
\hline ALVAL severity & Absent & Mild & Moderate & Severe \\
\hline Total number of patients & 50 & 50 & 47 & 29 \\
\hline Follow up (years) & $7(3-15)$ & $6(2-13)$ & $6(1-12)$ & $4(1-12)$ \\
\hline Age (range) & $57(29-79)$ & $57(25-85)$ & $58(41-76)$ & $59(36-74)$ \\
\hline$\%$ male patients & $54 \%(27: 50)$ & $42 \%(21: 39)$ & $43 \%(20: 27)$ & $31 \%(9: 21)$ \\
\hline Resurfacings vs THRs & $38 \%(19: 31)$ & $54 \%(27: 23)$ & $52 \%(25: 23)$ & $38 \%(11: 29)$ \\
\hline BMI & 26.7 & 26.4 & 24.3 & 25.5 \\
\hline Median (range) Co $(\mu \mathrm{g} / \mathrm{l})$ & $7.2(0.7-271)$ & $7.2(0.9-138.7)$ & $8.0(1.3-178.8)$ & $\begin{array}{c}9.1(1.8- \\
137.5)\end{array}$ \\
\hline Median (range) $\mathrm{Cr}(\mu \mathrm{g} / \mathrm{l})$ & $6.6(0.8-69.8)$ & $6.8(0.7-108.4)$ & $8.0(1.1-57.9)$ & $\begin{array}{c}7.7(1.9- \\
67.1)\end{array}$ \\
\hline $\begin{array}{l}\text { Mean annual volumetric } \\
\text { wear rate ( } \mathrm{mm}^{3} / \text { year) }\end{array}$ & $2.00(1.0-96.0)$ & $2.60(0.7-36.0)$ & $2.78(0.6-36.0)$ & $\begin{array}{c}3.40(0.8- \\
84.0)\end{array}$ \\
\hline
\end{tabular}


Extended data table 5. Demographics and clinical details of the training and validation datasets.

\begin{tabular}{|l|c|c|}
\hline & Data set 1 (training) & Data set 2 (test) \\
\hline Total number of patients & 405 & 10 (1- 20) \\
\hline Follow up (years) & $10(1-17)$ & $55(25-81)$ \\
\hline Age (range) & $56(25-85)$ & $(66 \%) 133: 68$ \\
\hline \% male patients & $(65 \%) 264: 141$ & $(77 \%) 154: 47$ \\
\hline Resurfacings vs THRs & $(78 \%) 314: 91$ & $21 \%(43: 148)$ \\
\hline \% patients with bilateral prostheses & $26 \%(105: 300)$ & $104 / 86 / 11$ \\
\hline UK/US/Australia & $216 / 173 / 16$ & $30 \%(60)$ \\
\hline \% failed & $28 \%(115)$ & $22.3 \%(45 / 201)$ \\
\hline \% mild/moderate/severe ALVAL & $20.2 \%(82 / 405)$ & 26.8 \\
\hline BMI & $2.0(0.1-271.0)$ & $(0.1-137.5)$ \\
\hline Median (range) Co ( $\mu$ g/l) & & \\
\hline Median (range) Cr ( $\mu g / l)$ & & \\
\hline
\end{tabular}


Extended data table 6. This table 1 shows the results of performance evaluation of the presented models on the test set. Taper-dominated wearing THRs were excluded from the test set for the ALVAL pre-operative model to better fit the clinical context in which this model would be exposed to. As the resulting test sets were different for pre-operative and post-operative models, their results are not directly comparable. Similarly high performance was observed across each of the models.

\begin{tabular}{|c|c|c|}
\hline Variable & ALVAL (pre-operative) & ALVAL (post-operative) \\
\hline Model & $\begin{array}{l}\text { Gradient boosted survival } \\
\text { analysis (regression tree based } \\
\text { learner; Cox-PH loss function) }\end{array}$ & $\begin{array}{l}\text { Gradient boosted survival } \\
\text { analysis (regression tree based } \\
\text { learner; Cox-PH loss function) }\end{array}$ \\
\hline Test data (n) & $\begin{array}{l}\text { Blinded test set excluding taper } \\
\text { wear dominated THRs (184) }\end{array}$ & Blinded test set (201) \\
\hline $\begin{array}{l}\text { Uno's C-index } \\
\qquad(95 \% \mathrm{Cl})\end{array}$ & $0.862(0.806-0.912)$ & $0.862(0.809-0.908)$ \\
\hline $\begin{array}{c}\text { IBS } \\
(95 \% \mathrm{CI})\end{array}$ & $0.062(0.043-0.083)$ & $0.066(0.047-0.087)$ \\
\hline $\begin{array}{c}\text { Mean AUROC(t) } \\
(95 \% \mathrm{Cl})\end{array}$ & $0.915(0.868-0.953)$ & $0.915(0.879-0.946)$ \\
\hline $\mathrm{ICl}$ at $\mathrm{T} \leq \mathrm{t}$ years & & \\
\hline$t=2$ & 0.005 & 0.011 \\
\hline$t=3$ & 0.009 & 0.016 \\
\hline$t=4$ & 0.019 & 0.023 \\
\hline$t=5$ & 0.023 & 0.019 \\
\hline$t=6$ & 0.029 & 0.016 \\
\hline$t=7$ & 0.024 & 0.013 \\
\hline
\end{tabular}




\begin{tabular}{|c|c|c|}
\hline $\mathbf{t}=\mathbf{8}$ & 0.020 & 0.023 \\
\hline $\mathbf{t}=\mathbf{9}$ & 0.017 & 0.051 \\
\hline $\mathbf{t}=\mathbf{1 0}$ & 0.018 & 0.031 \\
\hline
\end{tabular}

Extended data table 7. Cox proportional hazards modelling with all 606 patients involved in the study included. Patients were censored based on a minimum ALVAL grade of "mild" and above, then a second model was constructed with patients censored only if they had "moderate" ALVAL and above.

\begin{tabular}{|c|c|c|c|c|c|c|}
\hline \multicolumn{7}{|c|}{ Model 1: Survival based on ALVAL severity of mild, moderate or severe } \\
\hline Variable & Coeff & $\begin{array}{c}\text { Standard } \\
\text { error }\end{array}$ & $P$ value & $\begin{array}{l}\text { Hazard } \\
\text { ratio } \\
\text { (HR) }\end{array}$ & $\begin{array}{c}\text { HR } \\
\text { Lower Cl } \\
(95 \%)\end{array}$ & $\begin{array}{c}\text { HR } \\
\text { Upper C } \\
(95 \%)\end{array}$ \\
\hline Rank binding affinity for NTS & -1.463 & 0.404 & $<0.001$ & 0.232 & 0.105 & 0.511 \\
\hline $\begin{array}{l}\text { Log normalised cobalt } \\
\text { concentration }\end{array}$ & 1.649 & 0.136 & $<0.001$ & 5.202 & 3.982 & 6.797 \\
\hline Age & 0.005 & 0.011 & 0.667 & 1.005 & 0.984 & 1.026 \\
\hline Sex-M & -0.571 & 0.190 & 0.003 & 0.565 & 0.389 & 0.820 \\
\hline Type-THR & 0.779 & 0.201 & $<0.001$ & 2.180 & 1.471 & 3.230 \\
\hline \multicolumn{7}{|c|}{ Model 2: Survival based on ALVAL severity of moderate or severe } \\
\hline Rank binding affinity for NTS & -2.532 & 0.544 & $<0.0001$ & 0.079 & 0.027 & 0.231 \\
\hline $\begin{array}{l}\text { Log normalised cobalt } \\
\text { concentration }\end{array}$ & 1.656 & 0.178 & $<0.0001$ & 5.236 & 3.696 & 7.417 \\
\hline Age & 0.013 & 0.014 & 0.359 & 1.013 & 0.986 & 1.041 \\
\hline Sex-M & -0.631 & 0.247 & 0.011 & 0.532 & 0.328 & 0.863 \\
\hline Type-THR & 0.728 & 0.264 & 0.006 & 2.070 & 1.235 & 3.470 \\
\hline
\end{tabular}


Extended data figure 1. Time dependent $A U R O C(t)$ for the pre-operative model from two to ten years after implantation. The shaded area represents the $95 \%$ confidence intervals calculated via the Bootstrap method.

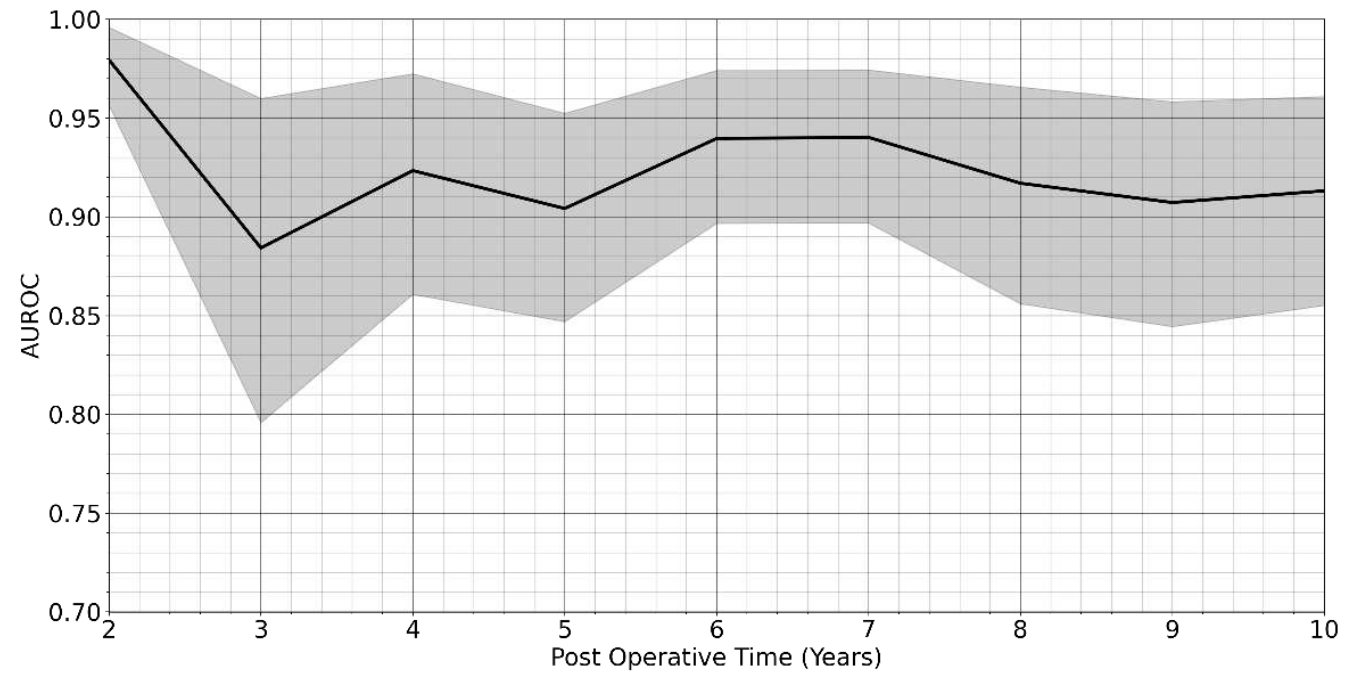

Extended data figure 2. Time dependent AUROC(t) for the post-operative model. The shaded area represents the $95 \%$ confidence intervals calculated via the Bootstrap method.

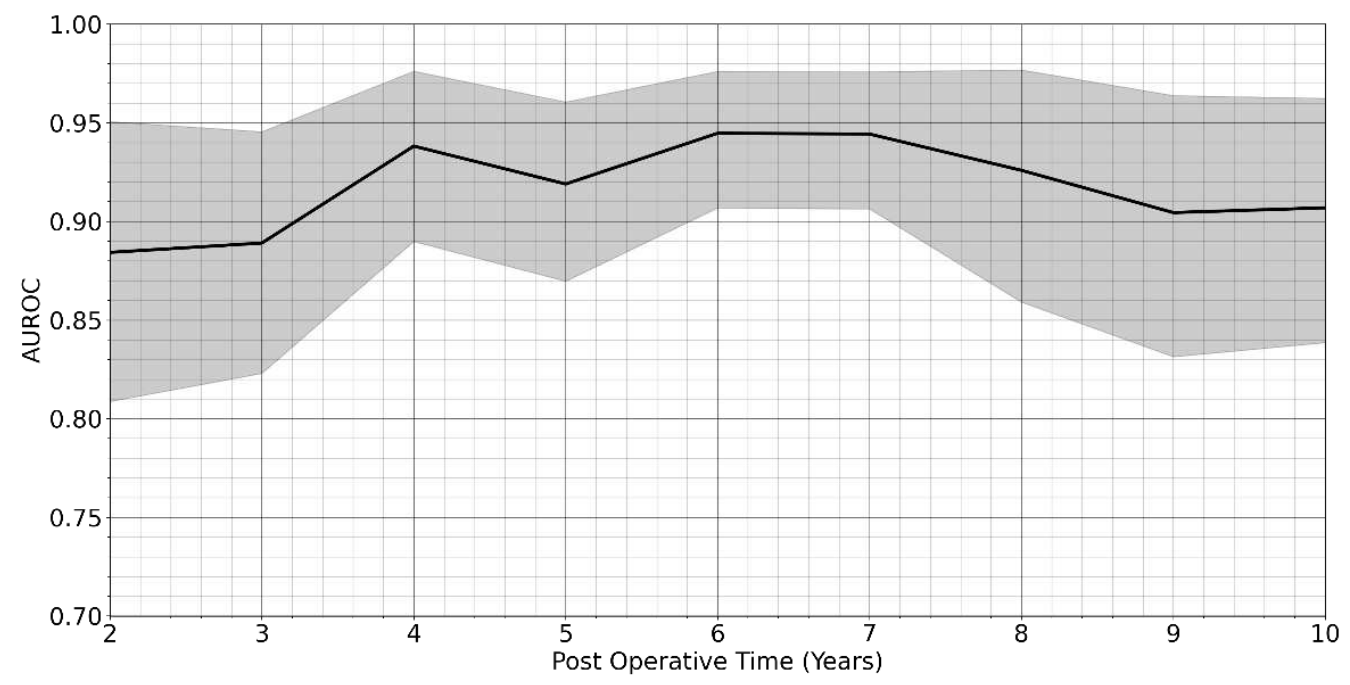




\section{Supplementary data}

Allele frequency comparisons - Excel spreadsheet 


\section{Supplementary Files}

This is a list of supplementary files associated with this preprint. Click to download.

- SupplementarydatafileAllelefrequencydistributionscombinedanalysis.xlsx 